\title{
TBX20 and the PROK2-PROKR1 pathway-new kid on the block in angiogenesis research
}

\author{
Michael Lichtenauer ${ }^{1}$, Christian Jung ${ }^{2}$ \\ ${ }^{1}$ Department of Internal Medicine II, Division of Cardiology, Paracelsus Medical University, Salzburg, Austria; ${ }^{2}$ Division of Cardiology, \\ Pulmonology, and Vascular Medicine, Medical Faculty, University Duesseldorf, Duesseldorf, Germany \\ Correspondence to: Christian Jung, MD, PhD. Division of Cardiology, Pulmonary Diseases and Vascular Medicine, Department of Medicine, \\ University Hospital Düsseldorf, 40225 Düsseldorf, Germany. Email: Christian.Jung@med.uni-duesseldorf.de. \\ Provenance: This is an invited Editorial commissioned by Guest Section Editor Yue Liu (The First Affiliated Hospital, Harbin Medical University, \\ Harbin, China). \\ Comment on: Meng S, Gu Q, Yang X, et al. TBX20 Regulates Angiogenesis Through the PROK2-PROKR1 Pathway. Circulation 2018;138:913-28.
}

Submitted Aug 13, 2018. Accepted for publication Aug 28, 2018.

doi: $10.21037 /$ atm.2018.08.41

View this article at: http://dx.doi.org/10.21037/atm.2018.08.41

\section{Introduction}

The term angiogenesis stems from the Greek expression for "angeīon" (vessel) and "genesis" (formation, development) and describes the formation of new blood vessels. Technically speaking, angiogenesis means the formation and sprouting of blood vessels from pre-existing vascular structures. By comparison, vasculogenesis is the de novo development of blood vessels from angioblasts (1). During embryogenesis, initial vascular structures are formed through vasculogenesis, which is followed by angiogenesis for further organ development and also blood vessel formation under physiological and pathophysiological conditions (such as tissue growth, wound healing, the female reproductive cycle, but also in cancer and ischemia). Angiogenesis is considered a pivotal process in almost all tissues in health and disease (2). The first step to induce angiogenesis is associated in most cases with pathological events and/or activating stimuli. Angiogenesis is a dynamic process that requires endothelial cells to transition from a resting to an activated state. This step involves the formation of a new vascular sprout and, once this is accomplished, the newly formed vascular structure is elongating. The subsequent process of further sprouting is a tightly regulated action of proliferation (3), cell migration (4), degradation of basement membranes to facilitate further expansiveness, vessel lumen formation, and last but not least also tissue stabilization (5). However, endothelial cells are just a part of the whole picture, as angiogenesis is further regulated by growth factors (e.g., vascular endothelial growth factor, VEGF), cytokines, chemokines, metalloproteinases, and on an intracellular level by activation of signaling kinases and gene transcription (6). Here in this editorial, we want to focus on the TBX20Prokineticin 2 (PROK2)-prokineticin receptor (PROKR1) signaling pathway.

\section{The study by Meng et al.}

In the recently published study by Meng et al., the authors sought to analyse the function of the key transcription factor T-box 20 (TBX20) and the signaling pathway which is activated during angiogenesis (7). TBX20 is considered a pivotal transcription factor during embryogenesis. Furthermore, it has been shown that its deficiency is associated with congenital heart disease. However, very little is yet known about its role in angiogenesis and vessel formation. Meng et al. determined the role of TBX20 in angiogenesis in a very elaborate approach using lossand gain-of-function assays by use of in vitro and in vivo experiments. TBX20 was shown to be an important regulator in cardiac development and knockout of Tbx20 in mice resulted in cardiac defects and embryonic lethality, whereas in human, mutations in the TBX20 gene are associated with different cardiac abnormalities (septation 


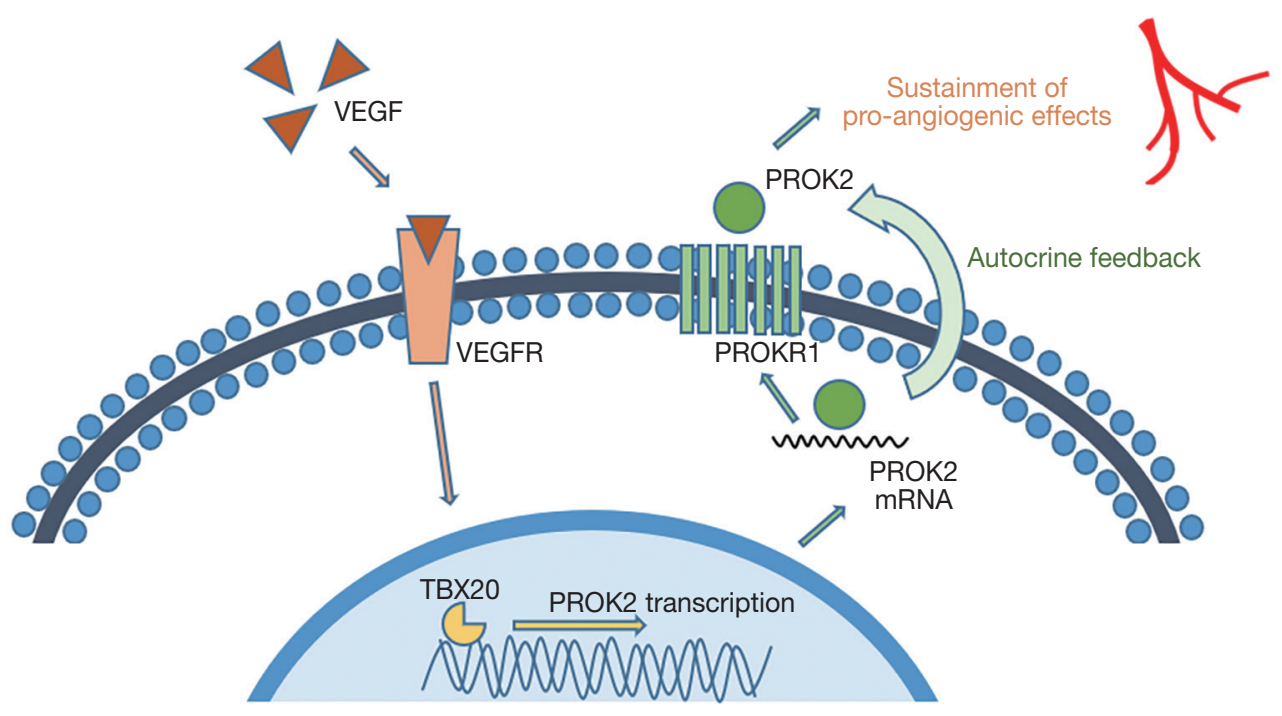

Figure 1 Illustrative model of the VEGF-TBX20-PROK2-PROKR1 signalling axis. Based on the data shown by Meng et al., VEGF and its binding to the VEGF receptor (VEGFR) triggers TBX20 activation and PROK2 transcription. PROK2 that acts on its receptor PROKR1 in an autocrine fashion. The authors presume that this mechanism might help to further sustain the pro-angiogenic signalling [adapted from Meng et al. (7)].

defects, altered chamber formation and cardiomyopathy) (8). A gene array was used to find key downstream targets of TBX20, which identified TBX20 as a novel transcription factor for signaling through the PROK2-PROKR1 pathway in the regulation of angiogenesis. Furthermore, the consequences of TBX20 activation or blocking were analysed in three different in vivo models. By application of a murine model of angiogenesis using subcutaneously implanted matrigel plugs saturated with VEGF, the authors observed that TBX20 deficiency led to reduced expression of PROK2 and also hindered angiogenesis. Additionally, a murine model of hind limb ischemia (induced by ligation of the femoral artery) was utilized to assess the effects of recombinant $\mathrm{PROK} 2$ administration. Here, enhanced angiogenesis and increased blood flow was documented in ischemic legs using Laser Doppler perfusion measurements. In a zebrafish model, the transient knockdown of TBX20 also resulted in impairment of angiogenesis.

In these experimental settings, the authors determined new and yet unknown functions of TBX20 and PROK2. PROK2, a secreted protein has been shown to influence angiogenesis, circadian rhythm, reproductive function and neurogenesis (9-11). Here in this study, the authors analyzed the whole TBX20-PROK2-PROKR1 axis and gained broad knowledge on its function and regulation in angiogenesis. Furthermore, they showed that TBX20 controls endothelial cell migration but not their proliferation. VEGF and its receptor VEGFR2 are known to be important regulators in angiogenesis. VEGFR2 activation by VEGF triggers downstream signaling cascades such as PI3K-Akt, PLC- $\gamma$-ERK1/2, SRC-Small GTPase and p38 (12,13). In this concise experimental setup by Meng et al. the TBX20-PROK2-PROKR1 axis, which can be activated by VEGF, joined these aforementioned pathways, as relevant alternative mediators in angiogenesis and can help to further sustain the pro-angiogenic effects of VEGF (see Figure 1).

\section{Future implications}

Angiogenesis is a fundamental process in both health and disease (14). Whereas considered beneficial as in wound healing and in ischemic diseases, overshooting angiogenesis can lead to cancer progression. Therefore, as angiogenesis itself is such a double-edged sword, a thorough knowledge of how it is regulated, initiated and deactivated is of paramount importance in order to better understand (patho-)physiological processes in many diseases.

Apart from angiogenesis, the understanding of intracellular signaling and its consequences are of utmost importance. As for angiogenesis, the same cellular signaling pathways (e.g., PI3K-Akt) are involved in cell activation 
and proliferation, which are beneficial effects in ischemia and tissue damage, and laterally reversed, of detrimental effects in oncogenesis. Yellon, Hausenloy and others have determined that activation of the ERK, Akt and PI3K kinases are involved in conditioning mechanisms in ischemia and can reduce the extent of tissue damage in myocardial infarction (15-18). On the other hand, the activation of the same signaling axes can lead to tumor progression in cancer $(19,20)$. Targeting single signaling factors of these pathways is thought to be an attractive strategy for the development of new drug compounds and novel therapeutic strategies to treat different forms of cancer. A fundamental knowledge of all these cross-linked signaling axes is of importance as intra- and inter-pathway compensatory loops exist in these pathways that can lead to the reactivation of trigger factors in the same cascade (upstream or downstream of the point of blockade). Moreover, as also underpinned by the data by Meng et al., activation of alternate signaling pathways can occur, which can hinder the effectiveness of cancer therapies.

Over recent years, our knowledge of molecular mechanisms in signaling axes has shown advances with a rapid increasing speed. Still, our understanding of how signaling factors interact and different pathways are interlocked with each other requires further research in order to provide the scientific background for the development of new drug agonist or antagonists to be applied in different disease conditions.

The data provided in this current experimental study provides a new jigsaw piece in the whole complex picture of intracellular signaling in angiogenesis. The TBX20PROK2-PROKR1 pathway described by Meng et al. could also be a therapeutic target to treat disease conditions that are associated with dysregulated angiogenesis. It could provide therapeutic angiogenesis in patients with ischemic heart failure, stroke or peripheral occlusive artery disease as PROK2 has been shown to improve blood perfusion and functional recovery.

\section{Acknowledgements}

None.

\section{Footnote}

Conflicts of Interest: The authors have no conflicts of interest to declare.

\section{References}

1. Risau W, Flamme I. Vasculogenesis. Annu Rev Cell Dev Biol 1995;11:73-91.

2. Duran CL, Howell DW, Dave JM, et al. Molecular Regulation of Sprouting Angiogenesis. Compr Physiol 2017;8:153-235.

3. Cavallo T, Sade R, Folkman J, et al. Ultrastructural autoradiographic studies of the early vasoproliferative response in tumor angiogenesis. Am J Pathol 1973;70:345-62.

4. Ausprunk DH, Folkman J. Migration and proliferation of endothelial cells in preformed and newly formed blood vessels during tumor angiogenesis. Microvasc Res 1977;14:53-65.

5. Senger DR, Davis GE. Angiogenesis. Cold Spring Harb Perspect Biol 2011;3:a005090.

6. Bosisio D, Salvi V, Gagliostro V, et al. Angiogenic and antiangiogenic chemokines. Chem Immunol Allergy 2014;99:89-104.

7. Meng S, Gu Q, Yang X, et al. TBX20 Regulates Angiogenesis Through the PROK2-PROKR1 Pathway. Circulation 2018;138:913-28.

8. Kirk EP, Sunde M, Costa MW, et al. Mutations in cardiac T-box factor gene TBX20 are associated with diverse cardiac pathologies, including defects of septation and valvulogenesis and cardiomyopathy. Am J Hum Genet 2007;81:280-91.

9. Kurebayashi H, Goi T, Shimada M, et al. Prokineticin 2 (PROK2) is an important factor for angiogenesis in colorectal cancer. Oncotarget 2015;6:26242-51.

10. Curtis VF, Wang H, Yang P, et al. A PK2/Bv8/PROK2 antagonist suppresses tumorigenic processes by inhibiting angiogenesis in glioma and blocking myeloid cell infiltration in pancreatic cancer. PLoS One 2013;8:e54916.

11. Ayari B, El Hachimi KH, Yanicostas C, et al. Prokineticin 2 expression is associated with neural repair of injured adult zebrafish telencephalon. J Neurotrauma 2010;27:959-72.

12. Karar J, Maity A. PI3K/AKT/mTOR Pathway in Angiogenesis. Front Mol Neurosci 2011;4:51.

13. Yu J, Bian D, Mahanivong C, et al. p38 Mitogen-activated protein kinase regulation of endothelial cell migration depends on urokinase plasminogen activator expression. J Biol Chem 2004;279:50446-54.

14. D'Alessio A, Moccia F, Li JH, et al. Angiogenesis and Vasculogenesis in Health and Disease. Biomed Res Int 
2015;2015:126582.

15. Hausenloy DJ, Yellon DM. New directions for protecting the heart against ischaemia-reperfusion injury: targeting the Reperfusion Injury Salvage Kinase (RISK)-pathway. Cardiovasc Res 2004;61:448-60.

16. Hausenloy DJ, Yellon DM. Reperfusion injury salvage kinase signalling: taking a RISK for cardioprotection. Heart Fail Rev 2007;12:217-34.

17. Wernly B, Goncalves I, Kiss A, et al. Differences in Stem Cell Processing Lead to Distinct Secretomes SecretionImplications for Differential Results of Previous Clinical Trials of Stem Cell Therapy for Myocardial Infarction. Biotechnol J 2017;12(9).

Cite this article as: Lichtenauer M, Jung C. TBX20 and the PROK2-PROKR1 pathway-new kid on the block in angiogenesis research. Ann Transl Med 2018;6(Suppl 1):S8. doi: 10.21037/atm.2018.08.41
18. Lichtenauer M, Mildner M, Hoetzenecker K, et al. Secretome of apoptotic peripheral blood cells (APOSEC) confers cytoprotection to cardiomyocytes and inhibits tissue remodelling after acute myocardial infarction: a preclinical study. Basic Res Cardiol 2011;106:1283-97.

19. Tian B, Chen X, Zhang H, et al. Urokinase plasminogen activator secreted by cancer-associated fibroblasts induces tumor progression via PI3K/AKT and ERK signaling in esophageal squamous cell carcinoma. Oncotarget 2017;8:42300-13.

20. Ciuffreda L, Incani UC, Steelman LS, et al. Signaling intermediates (MAPK and PI3K) as therapeutic targets in NSCLC. Curr Pharm Des 2014;20:3944-57. 